\title{
GENERALIZED REGULAR FUZZY BICLOSURE SPACES
}

\author{
U. D. Tapi, R. Navalakhe* \\ Department of Applied Mathematics \& Computational Science, Shri G. S. Institute of \\ Technology \& Science, Indore (M.P.) India. \\ *Corresponding address: sgsits.rachna@gmail.com \\ Received 17 October, 2011; Revised 21 August, 2012
}

\begin{abstract}
The purpose of this paper is to introduce new notion of regular fuzzy biclosure space called generalized regular fuzzy biclosure space and investigate some of their characterizations.
\end{abstract}

KEYWORDS : Fuzzy closure space, Fuzzy biclosure space, Generalized Regular fuzzy biclosure space.

MATHEMATICS SUBJECT CLASSIFICATION : 54A40.

\section{INTRODUCTION}

The concept of fuzzy sets and fuzzy set operations were first introduced by Zadeh in his classical paper [1]. Subsequently several authors have applied various basic concepts from general topology to fuzzy sets and developed the theory of fuzzy topological spaces. The notion of fuzzy sets naturally plays a very significant role in the study of fuzzy topology by Chang [2]. In 1970 N.Levine [3] introduced the concept of generalized closed sets as a generalization of closed sets to investigate some topological properties. Generalized closed (open) sets play an important role in general topology. Balasubramanian and Sundaram [4] defined generalized fuzzy closed sets in fuzzy topological spaces in order to extend some important properties of fuzzy closed sets to a larger family of sets . For instance, it was shown that compactness, normality and completeness in uniform space are inherited by generalized fuzzy closed subsets .

Closure spaces were introduced by E.Čech [5]. The notion of closure system and closure operator are very useful tools in several areas of classical mathematics. They play an important role in topological spaces , Boolean algebra, convex sets etc. This led several authors to investigate the closure operator in the frame work of fuzzy set theory. Gerla et al. [6] studied fuzzy closure operator and fuzzy closure system as extension of closure operator and closure system. Fuzzy closure spaces were first studied by A.S. Mashhour and M.H Ghanim [7].

Recently, Chawalit Boonpok [8] introduced the notion of biclosure spaces. Such spaces are equipped with two arbitrary closure operators. He extended some of the standard results of separation axioms in closure space to biclosure space. Thereafter a large number of papers have been written to generalize the concept of closure space to biclosure space. The authors $[9,10]$ have introduced the notion of fuzzy biclosure spaces and generalized the concept of fuzzy closure space to fuzzy biclosure space. In this paper we introduce and study the concept of generalized regular fuzzy biclosure spaces and study some of their properties. 


\section{PRELIMINARIES}

Definition 2.1[11]. A function $u: I^{X} \rightarrow I^{X}$ defined on the family $I^{X}$ of all fuzzy sets of $X$ is called a fuzzy closure operator on $X$ and the pair $(X, u)$ is called fuzzy closure space, if the following conditions are satisfied

1) $u \phi=\phi$

2) $A \leq u(A)$ for all $\mathrm{A} \in \mathrm{I}^{\mathrm{X}}$.

3) $u(A \vee B)=u(A) \vee u(B)$ for all $A, B \in \mathrm{I}^{\mathrm{X}}$.

Definition 2.2[9]. Two maps $u_{1}$ and $u_{2}$ from power set to itself are called fuzzy biclosure operators if they satisfy the following properties :

(i) $u_{1} \phi=\phi$ and $u_{2} \phi=\phi$

(ii) $A \leq u_{1} A$ and $A \leq u_{2} A$ for all $A \in I^{X}$

(iii) $u_{1}(A \vee B)=u_{1} A \vee u_{1} B$ and $u_{2}(A \vee B)=u_{2} A \vee u_{2} B$ for all $A, B \in I^{X}$.

A structure $\left(X, u_{1}, u_{2}\right)$ is called fuzzy biclosure space.

Definition 2.3 [9]. Let $(X, u)$ be a fuzzy closure space. A subset $A$ of a fuzzy closure space $(X, u)$ is called generalized fuzzy closed briefly, $g$-fuzzy closed, if $u A \leq G$ whenever $G$ is a fuzzy open subset of $(X, u)$ with $A \leq G$. The complement of a $g$ - fuzzy closed set is called $g$ - fuzzy open.

Proposition 2.4. Let $(X, u)$ be a fuzzy closure space and let $(Y, v)$ be a fuzzy closed subset of $(X, u)$. If $F$ is a g-fuzzy closed subset of $(Y, v)$, then $F$ is a g-fuzzy closed subset of $(X, u)$.

Proposition 2.5. Let $(X, u)$ be a fuzzy closure space. If $F$ is a fuzzy closed subset of $(X, u)$ and $F^{\prime}$ is a g- fuzzy closed subset of $(X, u)$, then $F \wedge F^{\prime}$ is a generalized fuzzy closed subset of $(X, u)$.

Proposition 2.6. Let $\left\{\left(X_{\alpha}, u_{\alpha}\right): \alpha \in J\right\}$ be a family of fuzzy closure spaces and let $\beta \in J$. Then $F$ is a $g$-fuzzy closed subset of $\left(X_{\beta}, u_{\beta}\right)$ if and only if $F \times \prod_{\substack{\alpha \neq \beta \\ \alpha \in J}} X_{\alpha}$ is a $g$-fuzzy closed subset of $\prod_{\alpha \in J}\left(X_{\alpha}, u_{\alpha}\right)$.

Proposition 2.7. Let $\left\{\left(X_{\alpha}, u_{\alpha}\right): \alpha \in J\right\}$ be a family of fuzzy closure spaces and let $\beta \in J$. Then $G$ is a $g$ - fuzzy open subset of $\left(X_{\beta}, u_{\beta}\right)$ if and only if $G \times \prod_{\substack{\alpha \neq \beta \\ \alpha \in J}} X_{\alpha}$ is a $g$-fuzzy open subset of $\prod_{\alpha \in J}\left(X_{\alpha}, u_{\alpha}\right)$.Proposition 2.8. Let $\left\{\left(X_{\alpha}, u_{\alpha}\right): \alpha \in J\right\}$ be a family of fuzzy closure spaces .For each $\beta \in J$, let $\pi_{\beta}: \prod_{\alpha \in J} X_{\alpha} \rightarrow X_{\beta}$ be the projection map.Then

(i) If $F$ is a $g$-fuzzy closed subset of $\prod_{\alpha \in J}\left(X_{\alpha}, u_{\alpha}\right)$, then $\pi_{\beta}(\mathrm{F})$ is a $g$-fuzzy closed subset of $\left(X_{\beta}, u_{\beta}\right)$.

(ii) If $F$ is a $g$-fuzzy closed subset of $\left(X_{\beta}, u_{\beta}\right)$, then $\pi_{\beta}^{-1}(\mathrm{~F})$ is a $g$-fuzzy closed subset of $\prod_{\alpha \in J}\left(X_{\alpha}, u_{\alpha}\right)$. 
Definition 2.9[9]. Let $(X, u)$ and $(Y, v)$ be fuzzy closure spaces. A map $f:(X, u) \rightarrow(Y, v)$ is called $g$-fuzzy irresolute, if $f^{-1}(F)$ is a $g$-fuzzy closed subset of $(X, u)$ for every $g$-fuzzy closed subset $F$ of $(Y, v)$.

Clearly, a map $f:(X, u) \rightarrow(Y, v)$ is $g$-fuzzy irresolute if and only if $f^{-1}(G)$ is a $g$-fuzzy open subset of $(X, u)$ for every $g$-fuzzy open subset $G$ of $(Y, v)$.

Definition 2.10[9]. Let $\left(X, u_{1}, u_{2}\right)$ and $\left(Y, v_{1}, v_{2}\right)$ be fuzzy biclosure spaces and let $i \in\{1,2\}$. A map $f:\left(X, u_{1}, u_{2}\right) \rightarrow\left(Y, v_{1}, v_{2}\right)$ is called $i-g$ - fuzzy irresolute if the map $f:\left(X, u_{i}\right) \rightarrow\left(Y, v_{i}\right)$ is $g$ fuzzy irresolute. A map $f$ is called $g$ - fuzzy irresolute if $f$ is $i-g$-fuzzy irresolute for each $i \in\{1,2\}$.

\section{GENERALIZED REGULAR FUZZY BICLOSURE SPACES}

In this section we introduce the concept of generalized regular fuzzy biclosure spaces and study some of their properties.

Definition 3.1. A fuzzy biclosure space $\left(X, u_{1}, u_{2}\right)$ is said to be generalized regular fuzzy biclosure space, briefly $g$ - regular fuzzy biclosure space if, for any fuzzy closed subset $F$ of $\left(X, u_{1}\right)$ and any fuzzy singleton $x \in 1-F$, there exist $g$ - fuzzy open subsets $U$ and $V$ of $\left(X, u_{2}\right)$ such that $x \in U$, $F \leq V$ and $U \wedge V=\phi$.

Remark 3.2. Every regular fuzzy biclosure space is $g$ - regular fuzzy biclosure space .

Example 3.3. Let $X=\{a, b\}$. For any $A \in I^{X}$, let supp $A=\{x \in X: A(x)>0\}$.Define fuzzy closure operators $u_{1}, u_{2}: I^{X} \rightarrow I^{X}$ by the following (for simplicity, we identify each ordinary subset of $X$ with its characteristic function) :

$$
\begin{array}{cc}
u_{1} A=u_{1} X=X & \text { if supp } A=X \\
u_{2} A=u_{2} X=X & \text { if supp } A=X \\
u_{1} A=u_{2} A=\phi & \text { if supp } A=\phi
\end{array}
$$

Then $\left(X, u_{1}, u_{2}\right)$ is a $g$ - regular fuzzy biclosure space .

Proposition 3.4. Let $\left(X, u_{1}, u_{2}\right)$ be fuzzy biclosure space. If $\left(X, u_{1}, u_{2}\right)$ is a $g$-regular fuzzy biclosure space, then for any $x \in X$ and every fuzzy open subset $U$ of $\left(X, u_{1}, u_{2}\right)$ containing $x$ there exists a $g$ fuzzy open subset $V$ of $\left(X, u_{2}\right)$ containing $x$ such that $u_{2} V \leq U$.

Proof . Suppose that $\left(X, u_{1}, u_{2}\right)$ is $g$-regular fuzzy biclosure space. Let $U$ is a fuzzy open subset of $\left(X, u_{1}, u_{2}\right)$ such that $x \in U$. Then $1-U$ is a fuzzy closed subset of $\left(X, u_{1}\right)$ which does not contain $x$.Therefore, there exist $g$ - fuzzy open subsets $W$ and $V$ of $\left(X, u_{2}\right)$ such that $1-U \leq W, x \in V$ and $W \wedge V=\phi$. Since $1-U \leq W$, we have $1-W \leq U$. Further $W \wedge V=\phi$ implies $V \leq 1-W \leq U$. Since $1-W$ is a $g$ - fuzzy closed subset of $\left(X, u_{2}\right)$ and $U$ is a fuzzy open subset of $\left(X, u_{2}\right)$, $u_{2}(1-W) \leq U$. Hence, $u_{2} V \leq u_{2}(1-W) \leq U$.

The following statement immediately follows Proposition 3.4 :

Corollary 3.5. Let $\left(X, u_{1}, u_{2}\right)$ be fuzzy biclosure space. If $\left(X, u_{1}, u_{2}\right)$ is g- regular fuzzy biclosure space, then for any $x \in X$ and every fuzzy closed subset $F$ of $\left(X, u_{1}, u_{2}\right)$ such that $x \notin F$, there exists a $g$ fuzzy open subset $G$ of $\left(X, u_{2}\right)$ such that $x \in G$ and $u_{2} G \wedge F=\phi$. 
Lemma 3.6. Let $\left(X, u_{1}, u_{2}\right)$ be a fuzzy biclosure space and let $\left(Y, v_{1}, v_{2}\right)$ be a fuzzy closed subspace of $\left(X, u_{1}, u_{2}\right)$. If $F$ is a fuzzy closed subset of $\left(Y, v_{1}, v_{2}\right)$, then $F$ is a fuzzy closed subset of $\left(X, u_{1}, u_{2}\right)$.

Lemma 3.7. Let $\left(X, u_{1}, u_{2}\right)$ be a fuzzy biclosure space and let $\left(Y, v_{1}, v_{2}\right)$ be a fuzzy closed subspace of $\left(X, u_{1}, u_{2}\right)$. If $G$ is both a $g$-fuzzy open subset of $\left(X, u_{1}\right)$ and $\left(X, u_{2}\right)$, then $G \wedge Y$ is both a $g$ fuzzy open subset of $\left(Y, v_{1}\right)$ and $\left(Y, v_{2}\right)$.

Proposition 3.8. Let $\left(X, u_{1}, u_{2}\right)$ be fuzzy biclosure space and let $\left(Y, v_{1}, v_{2}\right)$ be a fuzzy closed subspace of $\left(X, u_{1}, u_{2}\right)$. If $\left(X, u_{1}, u_{2}\right)$ is $g$-regular fuzzy biclosure space, then $\left(Y, v_{1}, v_{2}\right)$ is a $g$-regular fuzzy biclosure space.

Proof. Let $F$ be a fuzzy closed subset of $\left(Y, v_{1}\right)$ such that $y \notin F$. By Lemma $3.6 \quad F$ is a fuzzy closed subset of $\left(X, u_{1}\right)$ such that $y \notin F$.Since $\left(X, u_{1}, u_{2}\right)$ is $g$ - regular fuzzy biclosure space, there exist $g$ fuzzy open subsets $U$ and $V$ of $\left(X, u_{2}\right)$ such that $y \in U, F \leq V$ and $U \wedge V=\phi$.Consequently, $y \in U \wedge Y$ and $F \leq V \wedge Y$. By Lemma 3.7, $U \wedge Y$ and $V \wedge Y$ are $g$-fuzzy open subsets of $\left(Y, v_{2}\right)$ such that $(U \wedge Y) \wedge(V \wedge Y)=\phi$.Hence $\left(Y, v_{1}, v_{2}\right)$ is $g$-regular fuzzy biclosure space.

Proposition 3.9. Let $\left\{\left(X_{\alpha}, u_{\alpha}^{1}, u_{\alpha}^{2}\right): \alpha \in J\right\}$ be a family of fuzzy biclosure spaces. Then $\prod_{\alpha \in J}\left(X_{\alpha}, u_{\alpha}^{1}, u_{\alpha}^{2}\right)$ is $g$-regular fuzzy biclosure space if and only if $\left(X_{\alpha}, u_{\alpha}^{1}, u_{\alpha}^{2}\right)$ is $g$ - regular fuzzy biclosure space for each $\alpha \in J$.

Proof . Suppose that $\prod_{\alpha \in J}\left(X_{\alpha}, u_{\alpha}^{1}, u_{\alpha}^{2}\right)$ is $g$ - regular fuzzy biclosure space. Let $\beta \in J$ and let $F$ be fuzzy closed subset of $\left(X_{\beta}, u_{\beta}^{1}\right)$ such that $x_{\beta} \notin F$. Then $F \times \prod_{\substack{\alpha \neq \beta \\ \alpha \in J}} X_{\alpha}$ is a fuzzy closed subset of $\prod_{\alpha \in J}\left(X_{\alpha}, u_{\alpha}^{1}\right)$ such that $\left(x_{\alpha}\right)_{\alpha \in J} \notin F \times \prod_{\substack{\alpha \neq \beta \\ \alpha \in J}} X_{\alpha}$. Since $\prod_{\alpha \in J}\left(X_{\alpha}, u_{\alpha}^{1}, u_{\alpha}^{2}\right)$ is a $g$-regular fuzzy biclosure space, there exist $g$-fuzzy open subsets $U$ and $V$ of $\left(X_{\beta}, u_{\beta}^{2}\right)$ such that $x_{\beta} \in U, F \leq V$ and $U \wedge V=\phi$. Hence, $\left(X_{\beta}, u_{\beta}^{1}, u_{\beta}^{2}\right)$ is $g$-regular fuzzy biclosure space .

Conversely, Suppose that $\left(X_{\alpha}, u_{\alpha}^{1}, u_{\alpha}^{2}\right)$ is $g$-regular fuzzy biclosure space for each $\alpha \in J$. Let $F$ be fuzzy closed subset of $\prod_{\alpha \in J}\left(X_{\alpha}, u_{\alpha}^{1}\right)$ such that $\left(x_{\alpha}\right)_{\alpha \in J} \notin F$. Then $\pi_{\beta}(F)$ is a fuzzy closed subset of $\left(X_{\beta}, u_{\beta}^{1}\right)$ such that $x_{\beta} \notin \pi_{\beta}(F)$.Since $\left(X_{\beta}, u_{\beta}^{1}, u_{\beta}^{2}\right)$ is a $g$-regular fuzzy biclosure space there exist $g$-fuzzy open subsets $U$ and $V$ of $\left(X_{\beta}, u_{\beta}^{2}\right)$ such that $x_{\beta} \in U, F \leq V$ and $U \wedge V=\phi$. Therefore, $\left(x_{\alpha}\right)_{\alpha \in J} \in U \times \prod_{\substack{\alpha \neq \beta \\ \alpha \in J}} X_{\alpha}$ and $F \leq \pi_{\beta}^{-1}(V)=V \times \prod_{\substack{\alpha \neq \beta \\ \alpha \in J}} X_{\alpha}$.Consequently, $U \times \prod_{\substack{\alpha \neq \beta \\ \alpha \in J}} X_{\alpha}$ and $V \times \prod_{\substack{\alpha \neq \beta \\ \alpha \in J}} X_{\alpha}$ are $g$-fuzzy open subsets of $\prod_{\alpha \in J}\left(X_{\alpha}, u_{\alpha}^{2}\right)$ such that $\left(\left(U \times \prod_{\substack{\alpha \neq \beta \\ \alpha \in J}} X_{\alpha}\right) \wedge\left(V \times \prod_{\substack{\alpha \neq \beta \\ \alpha \in J}} X_{\alpha}\right)=\phi\right.$. Hence, $\prod_{\alpha \in J}\left(X_{\alpha}, u_{\alpha}^{1}, u_{\alpha}^{2}\right)$ is $g$-regular fuzzy biclosure space. 
Proposition 3.10. Let $\left(X, u_{1}, u_{2}\right)$ and $\left(Y, v_{1}, v_{2}\right)$ be fuzzy biclosure spaces. Let $f:\left(X, u_{1}, u_{2}\right) \rightarrow\left(Y, v_{1}, v_{2}\right)$ be injective fuzzy closed and $g$-fuzzy irresolute. If $\left(Y, v_{1}, v_{2}\right)$ is $g$-regular fuzzy biclosure space, then $\left(X, u_{1}, u_{2}\right)$ is a $g$-regular fuzzy biclosure space.

Proof . Let $F$ be fuzzy closed subset of $\left(X, u_{1}\right)$ such that $x \notin F$. Let $f(F)$ is a fuzzy closed subset of $\left(Y, v_{1}\right)$ such that $f(x) \notin f(F)$. Since $\left(Y, v_{1}, v_{2}\right)$ is $g$ - regular fuzzy biclosure space , there exist $g$-fuzzy open subsets $U$ and $\mathrm{V}$ of $\left(Y, v_{2}\right)$ such that $f(x) \in U, f(F) \leq V$ and $U \wedge V=\phi$. Consequently, $f^{-1}(U)$ and $f^{-1}(V)$ are $g$-fuzzy open subsets of $\left(X, u_{2}\right)$ such that $x \in f^{-1}(U)$, $F \leq f^{-1}(V)$ and $f^{-1}(U) \wedge f^{-1}(V)=\phi$. Hence, $\left(X, u_{1}, u_{2}\right)$ is $g$-regular fuzzy biclosure space.

\section{REFERENCES}

[1] Zadeh L A., Fuzzy sets, Information and control, 8(1965)338.

[2] Chang C L, Fuzzy Topological Spaces, Journal of mathematical Analysis and Applications, 24 (1968) 182.

[3] Levine N, Generalized closed sets in topology, Rend. Circ. Mat. Palermo, 19(1970) 2.

[4] Balasubramanian \& Sundaram P, On some generalization of fuzzy continuous functions, Fuzzy Sets and Systems, 86(1997) 93.

[5] Cech E, Topological Spaces, Interscience Publishers, John Wiley and Sons NewYork (1996).

[6] Biacino L \& Gerla G, An extension principle for closure operators, J. Math. Anal. Appl.,198(1996)1.

[7] Mashhour A S and Ghanim M H, Fuzzy closure spaces, Journal of Mathematical Analyze and Applications,106 (1985)154 .

[8] Boonpok C ,Hausdorff Biclosure Spaces, Int. J. Contemp. Math Sciences, 5(2010)359 .

[9] Tapi U D \& Navalakhe R, Fuzzy Biclosure Spaces, Int. Journal of Math. Analysis, 5(2011)789.

[10] Tapi U D \& Navalakhe R ,Generalized Normal Fuzzy Biclosure Spaces, International J. of Math. Sci. \& Engg. Appls (IJMSEA), 5( 2011)49.

[11] Ghanim M.H \& Fatma Al-Sirehy S, Topological modification of a fuzzy closure space, Fuzzy sets and systems, 27 (1988)211. 